CLNS-95/1369

LMU-TPW 95-16

hep-th/9510234

October 1995

\title{
On the Ubiquity of K3 Fibrations in String Duality
}

\author{
Paul S. Aspinwall \\ F.R. Newman Lab. of Nuclear Studies, \\ Cornell University, \\ Ithaca, NY 14853 \\ Jan Louis \\ Sektion Physik, \\ Universität München, \\ Theresienstrasse 37 , \\ D-80333 München, \\ Germany
}

\begin{abstract}
We consider the general case of $N=2$ dual pairs of type IIA/heterotic string theories in four dimensions. We show that if the type IIA string in this pair can be viewed as having been compactified on a Calabi-Yau manifold in the usual way then this manifold must be of the form of a K3 fibration. We also see how the bound on the rank of the gauge group of the perturbative heterotic string has a natural interpretation on the type IIA side.
\end{abstract}




\section{Introduction}

Recently, the quantum properties of $N=2$ supersymmetric field theories have been under active investigation. In particular, Seiberg and Witten [1] analyzed an asymptotically free $S U(2)$ Yang-Mills theory and determined the leading contribution of the low energy effective action for all values of the gauge coupling. It turns out that in the strong coupling region no massless $S U(2)$ gauge bosons exist; instead magnetic monopoles become massless and the effective low energy theory is best described by a weakly coupled dual $U(1)$ magnetic gauge theory.

These developments lead to intensive attempts to uncover the same physical phenomenon in string theory and study its gravitational and "stringy" generalizations. It has been conjectured that for strongly coupled heterotic $N=2$ vacua the "dual" description is provided by weakly coupled $N=2$ vacua of type II strings. This conjecture is supported by a number of concrete "pairs" of dual vacua [ 2, 3, 4, 5] where the perturbative effective action of the heterotic vacuum (at least, for the vector multiplets) has been matched with the dual type II vacuum in an appropriate expansion [ 2, 6, 7, 8, 9]. Furthermore Seiberg-Witten theory should appear in this context [10, 11] and can be recovered in the $\alpha^{\prime} \rightarrow 0$ limit [12].

These encouraging results make it interesting to go beyond a model by model analysis and study more generally the properties of the conjectured duality. However, for an arbitrary heterotic string vacuum it is presently unknown how to construct the dual partner. One might suspect that this duality in 4 dimensions is a consequence of string-string duality [ 13, 14, 15, 16] in six dimensions and the dual pairs of [3, 17] are indeed constructed in this way. It is not clear how to show the connection for the pairs of [2] however. Initial steps in this latter case were taken in [4] where it was noticed that both the type II string and the heterotic string could be "fibred" over a $\mathbb{P}^{1}$. That is, the Calabi-Yau manifold, $X$, on which the type IIA string was compactified could be written as a fibre bundle with base $\mathbb{P}^{1}$ and generic fibre a K3 surface where the heterotic string was compactified on $\mathrm{K} 3 \times T^{2}$ which can be written as a bundle with base $\mathbb{P}^{1}$ and generic fibre $T^{4}$. It is then tempting to try to use string-string duality fibre-wise to map the type IIA string on the K3 surface into a heterotic string on $T^{4}$.

The relevance of K3-fibrations was first noticed in [7] where it was shown that such a structure fits nicely with properties of the dual heterotic string compactified on $\mathrm{K} 3 \times T^{2}$. (Various related aspects of K3-fibrations were also discussed in [ 18, 19].) In this paper we will take this point further and argue that the appearance of the K3-fibration is actually unavoidable. The argument will borrow results from the "phase" picture of the moduli space of $N=2$ conformal field theories [ 20, 21]. In particular, we need to understand clearly what we mean by an object that is recognizably a heterotic string and an object that is recognizably a type IIA string theory compactified on a Calabi-Yau manifold. In the latter case, we know from the phase picture that we need to identify a "Calabi-Yau" phase of the moduli space and we will see that we must also identify a "weakly-coupled" phase in 
the heterotic string moduli space.

The phase picture allowed a complete resolution of the question of Calabi-Yau manifolds without mirror partners [22]. We will see that exactly the same considerations apply to heterotic strings without dual type II partners and visa versa. If we demand that we do have a dual pair in which the weakly-coupled phase of a heterotic string maps into the Calabi-Yau phase of a type IIA string then we will see that this Calabi-Yau manifold must have a K3-fibration.

One bound which appears naturally on the heterotic side is on the rank of the gauge group. One can use conformal field theory considerations to show that it cannot exceed 24 . It has been troubling that no such bound is known on the type II side. We will see how K3-fibrations give a natural interpretation to this effect although it appears that this bound can be broken by nonperturbative effects on the heterotic side.

\section{Perturbative $N=2$ Heterotic String Vacua}

Let us start by summarizing the generic properties of perturbative heterotic $N=2$ vacua. The heterotic string is based on a conformal field theory with central charge $(c, \bar{c})=(26,15)$. Out of this total central charge a $(c, \bar{c})=(4,6)$ "block" is used to build the four-dimensional space-time while the left over $(c, \bar{c})=(22,9)$ conformal field theory is only further constrained by the amount of space-time supersymmetry and the size of the gauge group. $N=2$ spacetime supersymmetry requires that the right moving internal $\bar{c}=9$ conformal field theory splits into a free complex boson (of central charge $\bar{c}=3$ ) and a $\bar{c}=6$ conformal field theory with $N=4$ world-sheet supersymmetry [23]. The gauge group $G_{\text {het }}$ arises from the left moving $c=22$ conformal field theory and the free $\bar{c}=3$ boson. The latter gives rise to two Abelian $U(1)$ gauge bosons which are identified with the graviphoton and the vector partner of the dilaton. The $c=22$ conformal field theory generates an arbitrary (possibly non-Abelian) gauge group $G^{\prime}$ of maximal rank 22. Therefore $G_{\text {het }}=G^{\prime} \times[U(1)]^{2}$ and the rank of $G_{\text {het }}$ is bounded by

$$
2 \leq \operatorname{rank}\left(G_{\text {het }}\right) \leq 24
$$

The supersymmetric partners of the gauge bosons are two gauginos and a complex scalar all in the adjoint representation of $G_{\text {het }}$; they form what is called an $N=2$ vector multiplet. The matter fields usually transform in the fundamental representation of $G_{\text {het }}$ and they reside in $N=2$ hypermultiplets which contain two Weyl fermions and four real scalars. All string vacua also feature gauge neutral scalars termed moduli which are exactly flat directions of the effective potential and which parameterize the perturbative degeneracy of a given vacuum family. Such moduli arise as the scalars of either vector or hypermultiplets and they can be thought of as parameters taking values in a moduli space. $N=2$ supersymmetry implies that the moduli space $\mathscr{M}$ is always locally a product [24]

$$
\mathscr{M}=\mathscr{M}_{\mathrm{v}} \times \mathscr{M}_{\mathrm{h}},
$$


where $\mathscr{M}_{\mathrm{v}}\left(\mathscr{M}_{\mathrm{h}}\right)$ is spanned by the scalars in the vector multiplets (hypermultiplets).

The scalar fields $T^{i}, i=1, \ldots, r \equiv \operatorname{rank}\left(G^{\prime}\right) \leq 22$ in the Cartan subalgebra of $G^{\prime}$ are flat directions of the effective potential. If all $T^{i}$ s are turned on (a generic point in the moduli space) $G^{\prime}$ is broken to its maximal Abelian subgroup (which is at most $[U(1)]^{22}$ ) while at special points in the moduli space this gauge symmetry can be enhanced to a non-Abelian group.

In addition to the $T^{i}$ moduli there is also always the string dilaton present in the massless spectrum. Its vacuum expectation value determines the string coupling constant and organizes the string perturbation theory. Together with the dual axion of an antisymmetric tensor field it forms a complex scalar $S$ of an Abelian $U(1)$ vector multiplet $^{1}$ and in the standard heterotic normalization one defines

$$
S=\frac{1}{g^{2}}-i \frac{\theta}{8 \pi^{2}}
$$

such that large $S$ corresponds to weak coupling. With this convention the Peccei-Quinn symmetry associated with the axion is given by a shift

$$
\theta \rightarrow \theta+2 \pi r, \quad S \rightarrow S-\frac{i r}{4 \pi},
$$

where $r$ is a continuous parameter in string perturbation theory. However, once nonperturbative corrections are taken into account this continuous symmetry is broken down to a discrete subgroup and then $r$ must be an integer.

The couplings of the vector multiplets (the Kähler potential and the gauge couplings) are entirely encoded in a holomorphic prepotential $F_{0}$. Due to the non-renormalization theorem of $N=2$ supersymmetry $F_{0}$ does not receive quantum corrections beyond one-loop in perturbation theory but can (and is) corrected nonperturbatively. Using the fact that the dilaton counts string loops, $F_{0}$ can be expanded for large $S$ (weak coupling) according to

$$
F_{0}=F_{0}^{(0)}\left(S, T^{i}\right)+F_{0}^{(1)}\left(T^{i}\right)+F_{0}^{(n p)}\left(S, T^{i}\right)
$$

where $F_{0}^{(0)}\left(F_{0}^{(1)}\right)$ is the tree level (one-loop) contribution while $F_{0}^{(n p)}$ denotes nonperturbative corrections. The universal couplings of the dilaton and the continuous Peccei-Quinn symmetry of the axion completely determine $F_{0}^{(0)}$ for all heterotic string vacua to be [ 25, 26, 27, 28] (using the conventions of [28])

$$
F_{0}^{(0)}=-\gamma_{i j} T^{i} T^{j} S, \quad \gamma_{i j}=\operatorname{diag}(+,-, \ldots,-),
$$

where $S$ and $T^{i}$ are the "special" $N=2$ coordinates. $F_{0}^{(1)}\left(T^{i}\right)$ is necessarily $S$-independent. The tree level prepotential given in (6) corresponds to the Kähler potential

$$
K=-\log (S+\bar{S})-\log \gamma_{i j}\left(T^{i}+\bar{T}^{i}\right)\left(T^{j}+\bar{T}^{j}\right),
$$

\footnotetext{
${ }^{1}$ The $U(1)$ gauge bosons arises in the $\bar{c}=3$ block of the conformal field theory.
} 
which is the Kähler potential of the homogeneous space

$$
\mathscr{M}_{\mathrm{v}}^{0}=\frac{S U(1,1)}{U(1)} \times \frac{S O(2, r)}{S O(2) \times S O(r)},
$$

up to discrete identifications. Note that (8) is subject to string loop corrections and, in general, is not isomorphic to $\mathscr{M}_{\mathrm{v}}$.

So far we concentrated on the lowest order (two derivative) couplings in the effective action. There is a special class of higher derivative curvature couplings which arise from chiral integrals in $N=2$ superspace and therefore are also governed by holomorphic functions $F_{n}\left(S, T^{i}\right)$ of the vector multiplets. These are couplings of the form $g_{n}^{-2} R^{2} G^{2 n-2}$ where $R$ is the (anti-self-dual) Riemann tensor, $G$ is the field strength of the graviphoton and the couplings $g_{n}$ obey [29, 30, 31] ${ }^{2}$

$$
g_{n}^{-2}=\operatorname{Re} F_{n}\left(S, T^{i}\right)+\mathcal{A}_{n} .
$$

At the string tree level $\mathcal{A}_{n}=0$ holds and therefore $g_{n}^{-2}$ is a harmonic function of the vector moduli. However, at one-loop $g_{n}^{-2}$ develops a holomorphic anomaly $\mathcal{A}_{n}$. For $n=1$ one finds [32, 33, 34, 6]

$$
\partial_{i} \bar{\partial}_{\bar{\jmath}} g_{1}^{-2}=\frac{b}{16 \pi^{2}} \partial_{i} \bar{\partial}_{\bar{\jmath}} K, \quad b=2\left(n_{\mathrm{h}}-n_{\mathrm{v}}+23\right)
$$

where $K$ is the Kähler potential defined in $(7) ; n_{\mathrm{h}}\left(n_{\mathrm{v}}\right)$ counts the number of hypermultiplets (vector multiplets). Similar to (5) the holomorphic $F_{n}$ 's can be expanded in the dilaton according to ${ }^{3}$

$$
F_{n}=F_{n}^{(0)}\left(S, T^{i}\right)+F_{n}^{(1)}\left(T^{i}\right)+F_{n}^{(n p)}\left(S, T^{i}\right) .
$$

Again $F_{n}^{(1)}\left(T^{i}\right)$ is dilaton independent and the perturbative Peccei-Quinn symmetry of the axion determines

$$
F_{1}^{(0)}=24 S, \quad F_{n>1}^{(0)}=0,
$$

where the normalization of $F_{1}^{(0)}$ is fixed by the normalization of (10).

\section{The Dual Type IIA String}

Type II string vacua are built from conformal field theories of central charge $(c, \bar{c})=(15,15)$. The four-dimensional space-time degrees of freedom arise from a $(c, \bar{c})=(6,6)$ block leaving $(c, \bar{c})=(9,9)$ as the internal conformal field theory. The standard way to obtain $N=2$ spacetime supersymmetry is to demand a left-right symmetric $N=2$ world-sheet supersymmetry of the internal $(c, \bar{c})=(9,9)$ conformal field theory. ${ }^{4}$ An example of such a conformal field

\footnotetext{
${ }^{2}$ We slightly change notation here. In this paper $F_{n}$ always is a holomorphic quantity while $g_{n}$ is the non-harmonic coupling.

${ }^{3}$ We thank B. de Wit for discussions of this point.

${ }^{4}$ We do not consider asymmetric constructions [35] in this paper; some examples have recently been discussed in [0, 5].
} 
theory is provided by the non-linear $\sigma$-model on a Calabi-Yau manifold. Let us consider a type IIA superstring compactified on a Calabi-Yau manifold $X$. Equivalently, we can compactify the type IIB string on $Y$, the mirror of $X$.

The number of vector- and hypermoduli is directly related to the Hodge numbers of the Calabi-Yau compactification. Specifically one finds $n_{\mathrm{v}}=h^{1,1}(X), n_{\mathrm{h}}=h^{2,1}(X)+1$ for type IIA and $n_{\mathrm{v}}=h^{2,1}(Y), n_{\mathrm{h}}=h^{1,1}(Y)+1$ for type IIB; in both cases the additional hypermultiplet corresponds to the type II dilaton. The gauge group $G_{I I}$ always is a product of $\left(n_{\mathrm{v}}+1\right)$ Abelian $U(1)$ factors and therefore one has in general

$$
\operatorname{rank}\left(G_{I I}\right)=n_{\mathrm{v}}+1
$$

where the "+1" counts the graviphoton. The dilaton always is the member of a hypermultiplet and thus (2) implies that for type II vacua $\mathscr{M}_{\mathrm{v}}$ is independent on the dilaton and therefore determined at the string tree level exactly with no further corrections perturbatively or nonperturbatively.

Near the large radius limit of $X$, the position in the moduli space $\mathscr{M}_{\mathrm{v}}$ is given by the complexified Kähler form on $X$ - that is, an object of the form $B+i J \in H^{2}(X, \mathbb{C})$, where we expand

$$
B+i J=\sum_{\alpha=1}^{h^{1,1}}(B+i J)_{\alpha} e_{\alpha},
$$

so that $B_{\alpha}$ and $J_{\alpha}$ are real numbers and $e_{\alpha}$ represents a basis of $H^{2}(X, \mathbb{Z})$ (we assume that $\left.b_{1}(X)=0\right)$. As $H^{2}(X)$ is dual to $H_{2}(X)$ and $H_{2}(X)$ is dual to $H_{4}(X)$, we have an isomorphism between $H^{2}(X)$ and $H_{4}(X)$. Using this isomorphism, we can associate a divisor, $D_{\alpha}$, of $X$ to each $e_{\alpha}$.

In the large radius limit (or large complex structure limit for type IIB) the holomorphic prepotential has the generic structure ${ }^{5}$

$$
F_{0}=-\frac{i}{6} \sum_{\alpha, \beta, \gamma}\left(D_{\alpha} \cdot D_{\beta} \cdot D_{\gamma}\right) t_{\alpha} t_{\beta} t_{\gamma}+\cdots+\text { worldsheet instantons }
$$

where $t_{\alpha}=(B+i J)_{\alpha}$ denotes the moduli of the vector multiplets in $N=2$ special coordinates and we have omitted the $\sigma$-model loop terms.

Recall [36, 37] that the instanton corrections in (15) come from rational curves within $X$. As these curves get larger, the instanton effects become smaller. In order for (15) to make sense, we must assume that the sum produced by the instantons is convergent. If it is not, one can often replace the Calabi-Yau model by another theory (such as a Landau-Ginzburg theory) in which some other instanton sum converges. This is regarded as the underlying $N=2$ superconformal field theory being in another "phase" [ 20, 21]. If we insist that we

\footnotetext{
${ }^{5}$ We will use a dot to represent the intersection product between homology classes and also the natural pairing between homology and cohomology. We will also not distinguish between a divisor and its homology class.
} 
are in a Calabi-Yau phase and thus that (15) is a convergent series then we are demanding that all the rational curves on $X$ are sufficiently large.

Now we are interested in identifying those Calabi-Yau compactification which can serve as possible dual descriptions of heterotic vacua. The idea is, that once all nonperturbative effects have been taken into account, the heterotic theory and the type II theory describe exactly the same physics in the infrared. One test of the proposed duality is to check that both theories have identical moduli spaces. However, in either case we only know the respective weak coupling limits. Nevertheless due to the product structure of the moduli space and the fact that the dilaton resides in different super-multiplets in the two theories it is possible to compare the heterotic $\mathscr{M}_{\mathrm{v}}$ in a weak coupling expansion with the exact $\mathscr{M}_{\mathrm{v}}$ of the type II vacuum. In particular, one of the vector moduli $t_{\alpha}$ in the type IIA vacuum must be the image of the axion-dilaton pair of the heterotic string. Let us denote this particular member by $t_{s}=(B+i J)_{s}$ and let $D_{s}$ be the associated divisor. ${ }^{6}$ As we have just said we can only compare the moduli spaces for large $S$ and hence $t_{s}$ also has to be large. In this limit the type IIA $B_{s}$-field is a periodic variable and obeys $B_{s} \rightarrow B_{s}+1$. This is consistent with the Peccei-Quinn symmetry $\theta \rightarrow \theta+2 \pi$ of the heterotic axion if we identify

$$
(B+i J)_{s}=4 \pi i S
$$

Using the fact that both the complexified Kähler form and the axion-dilaton are special $N=2$ coordinates (16) is exact up to symplectic $S p\left(2 n_{\mathrm{v}}+2, \mathbb{Z}\right)$ reparametrizations and not only valid at the large radius limit.

The other vector multiplets will be denoted by $e_{i}$ (or equivalently $D_{i}$ ), for $i=1, \ldots, n_{\mathrm{v}}-1$. Given our assumption that the weakly coupled phase of the heterotic string maps into the Calabi-Yau phase of the type IIA string we can obtain from (6) and (15) that

$$
D_{s} \cdot D_{s} \cdot D_{s}=0, \quad D_{s} \cdot D_{s} \cdot D_{i}=0 \quad \forall i
$$

$D_{s} \cdot D_{s}$ must be an element of the second homology of $X$. Since (17) tells us that this element has zero intersection with any 4 -cycle, it must be trivial. That is,

$$
D_{s} \cdot D_{s}=0
$$

We know that $D_{s}$ itself is not trivial as the associated modulus is not trivial. In the language of algebraic geometry we have shown that the "numerical $D$-dimension" of the divisor $D_{s}$ is one.

Next, let us analyze the positivity properties of $D_{s}$. We have asserted that we are in the Calabi-Yau phase of the type IIA string. From the work of [ 38] it follows that we know something about $J$. Very roughly, it means that $J$ lies within the Kähler cone of $X$. That

\footnotetext{
${ }^{6}$ Strictly speaking we need to establish that $D_{s}$ is a "Q্Q-Cartier" divisor. This follows since an irrational class would not be compatible with the Peccei-Quinn symmetry.
} 
is, the volume of any algebraic subspace within $X$ is measured by the Kähler form to be positive. ${ }^{7}$ Suppose we take a theory which is in the Calabi-Yau phase and modify the Kähler form as

$$
J \rightarrow J+\lambda e_{s}
$$

where $\lambda$ is a large real number. In the heterotic language, this amounts to increasing the value of the dilaton, i.e., making the string more weakly coupled. Thus, the instanton sums will become more convergent. If this is to have the same effect in the type IIA string, then this change in the Kähler form must keep us inside the Kähler cone of $X$. This amounts to the assertion that

$$
e_{s} \cdot C \geq 0
$$

for any algebraic curve, $C$, within $X$. In the language of algebraic geometry, this tells us that $D_{s}$ is "nef".

Lastly we need one more property of $D_{s}$ which follows from identifying the higher derivative curvature couplings $F_{n}$ in both theories. Since the $F_{n}$ 's depend only on the vector multiplets they are independent on the type II dilaton and one finds that they arise solely at the $n$-loop order. In the large radius limit one obtains [ 29, 30]

$$
\begin{aligned}
F_{1} & =-\frac{4 \pi i}{12} \sum_{\alpha}\left(D_{\alpha} \cdot c_{2}\right) t_{\alpha}+\text { worldsheet instantons } \\
F_{n>1} & =\text { const. }+ \text { worldsheet instantons }
\end{aligned}
$$

where $c_{2}$ denotes the second Chern class of $X$. Furthermore, $g_{1}^{-2}$ develops a holomorphic anomaly given by [29]

$$
\partial_{\alpha} \bar{\partial}_{\bar{\beta}} g_{1}^{-2}=\frac{1}{6}\left(17+5 n_{\mathrm{v}}+n_{\mathrm{h}}\right) \partial_{\alpha} \bar{\partial}_{\bar{\beta}} K-R_{\alpha \bar{\beta}},
$$

where $R_{\alpha \bar{\beta}}$ is the Ricci-tensor on the moduli space. Let us first note that for $n>1$ the large $S$ limits of (12) and (21) already agree with no further conditions imposed. However, identifying the $F_{1}$ 's of the dual pairs results in a constraint on $c_{2}(X)$. Note that the holomorphic anomaly of (22) simplifies if, in the large $J$ limit, the type II prepotential and Kähler potential are identical with the heterotic $F_{0}$ and $K$ of (6) and (7). A straightforward computation shows that (22) reduces in this limit to

$$
\partial_{\alpha} \bar{\partial}_{\bar{\beta}} g_{1}^{-2}=\frac{b}{12} \partial_{\alpha} \bar{\partial}_{\bar{\beta}} K
$$

where $b$ is defined in (10). We see that the anomalies of a dual pair coincide up to the overall normalization. In turn this can be used to align the overall normalization between the $F_{1}$ 's

\footnotetext{
${ }^{7}$ Actually, there are quantum corrections to this statement and the walls of the classical Kähler cone have to be moved in a little to make sure the instanton sum remains finite. These corrections have no effect on our argument here.
} 


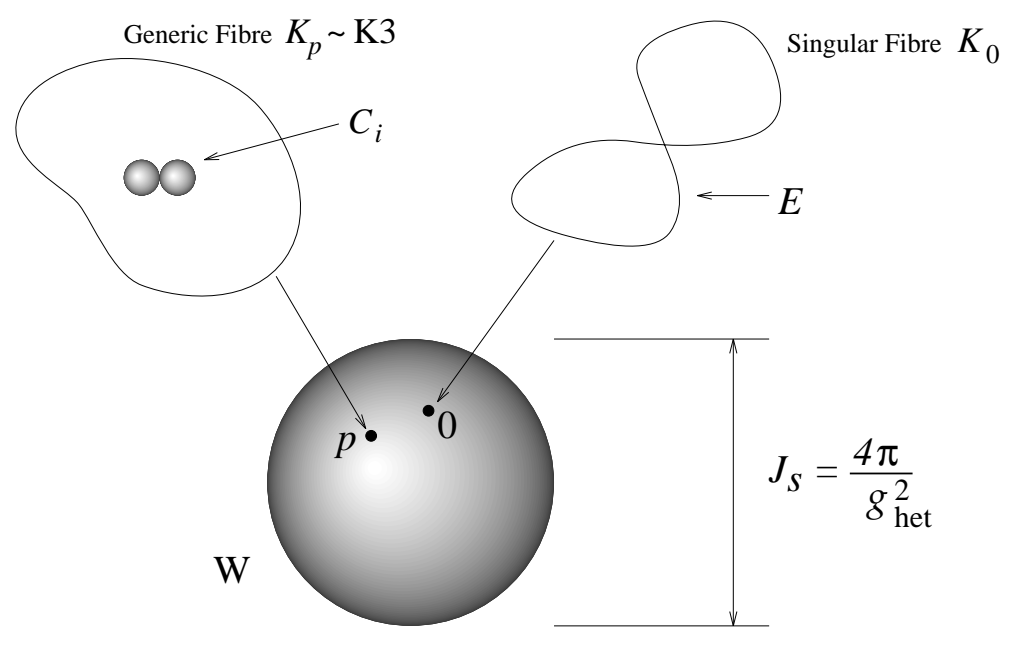

Figure 1: $X$ as a K3-fibration.

in each vacuum. Identifying the $F_{1}$ 's using (16), (12), (10), (23) and (21) results in

$$
D_{s} \cdot c_{2}(X)=24
$$

We may now use the results of Oguiso [39] which state that

Theorem 1 Let $X$ be a minimal Calabi-Yau threefold. Let $D$ be a nef divisor on $X$. If the numerical $D$-dimension of $D$ equals one and $D \cdot c_{2}(X)>0$ then there is a fibration $\Phi: X \rightarrow W$, where $W$ is $\mathbb{P}^{1}$ and the generic fibre is a K3 surface.

We have obtained the desired result $-X$ has to be a K3-fibration in order to be the dual partner of a heterotic vacuum. Note that in general the fibre is allowed to degenerate to something other than a K3 surface over a finite number of points on $W$. This fibration is shown in figure 1 .

We should emphasize two points about our derivation of the result. Firstly, we assumed that the Calabi-Yau manifold in the type IIA theory was in the Calabi-Yau phase when the heterotic string was weakly-coupled. It is certainly conceivable that there may be dual pairs where the phases are not aligned like this. In such an example, there is then no reason why a Calabi-Yau manifold on the type IIA side, which appears for some strongly-coupled regime of the heterotic moduli space, should be a K3-fibration. In other words, the weakly-coupled heterotic string might be dual to a type IIA string theory on a manifold $X$, which is not a K3-fibration, in the following sense. The instanton sum in (15) is necessarily divergent but could be reinterpreted in terms of some other non-Calabi-Yau phase (such as a perturbed Landau-Ginzburg theory or orbifold [21]) to give a convergent sum of corrections.

Secondly, we are stating only that the type IIA string is compactified on a K3-fibration. The type IIB string is compactified on $Y$, the mirror of $X$, which may or may not be a 
K3-fibration. The question as to what extent mirror symmetry acts within the class of K3-fibrations has not been analyzed at this point in time so we cannot make any strong statements about when $Y$ is a K3-fibration. One can observe though that in the cases discussed in [2], where the mirror map is in the Greene-Plesser orbifold form [ 40], then the mirror orbifolding operation preserves the fibration structure and so both $X$ and $Y$ are K3-fibrations.

Note that (24) not only tells us that $D_{s} \cdot c_{2}(X)>0$ but that the value is the Euler characteristic of a K3 surface. This tells us that the element in $H_{4}(X)$ corresponding to $D_{s}$ is given precisely by a generic K3 fibre [39]. Clearly the 2-cycle dual to this is given by $W$, the base space. It then follows that $J_{s}$ is the cohomology element that gives us the size of this 2-cycle. That is, the dilaton in the heterotic string is given by the size of the base $\mathbb{P}^{1}$ in the fibration of $X$.

Thus far we have made no appeal to string-string duality in six-dimensions but we can use this duality to reproduce the result we have just found concerning the dilaton. Let us set the axion in the heterotic string to zero for simplicity. In [15] it was shown that

$$
S_{6}=\frac{1}{S_{6}^{\prime}}, \quad S_{6} h_{6}=h_{6}^{\prime},
$$

where $S_{6}$ is the dilaton in six dimensions for the heterotic string and $h_{6}$ is its metric with the corresponding values primed for the type IIA string. Let us apply this to our picture in four dimensions by "compactifying" the six-dimensional space-time over $\mathbb{P}^{1}$. Given the Weyl-rescaling between the type IIA and heterotic space-times, the areas of these rational curves will differ by a factor of $S_{6}$. The action for the heterotic string can then be written

$$
\begin{aligned}
L_{\mathrm{het}} & =\int d^{6} x \sqrt{h_{6}} S_{6}(R+\ldots)=\operatorname{Area}\left(\mathbb{P}^{1}\right) \int d^{4} x \sqrt{h_{4}} S_{6}(R+\ldots) \\
& =\operatorname{Area}\left(\mathbb{P}^{1}\right)^{\prime} \int d^{4} x \sqrt{h_{4}}(R+\ldots) .
\end{aligned}
$$

This last form of the action tells us that the area of the base in the type IIA picture gives the four-dimensional dilaton of the heterotic string as we saw above.

For the dilaton modulus in the type IIB picture we can use the monomial-divisor mirror of [41] (or, more specifically, the results of [42, 43]) to find the corresponding deformation of complex structure of $Y$. Note also that we know the result exactly — since the dilaton is given by the Kähler form, the map between the dilaton and the complex modulus of $Y$ is corrected by solutions to the Picard-Fuchs equation.

For example, in [2] $Y$ was taken to be an orbifold of the hypersurface

$$
x_{1}^{12}+x_{2}^{12}+x_{3}^{6}+x_{4}^{6}+x_{5}^{2}-12 \psi x_{1} x_{2} x_{3} x_{4} x_{5}-2 \phi x_{1}^{6} x_{2}^{6}=0,
$$

in $\mathbb{P}_{\{1,1,2,2,6\}}^{4}$. It was then conjectured that there was a heterotic string dual to a type IIB string compactified on $Y$. From what we have said above and using the results of [42] it 
follows that the heterotic dilaton is given by

$$
S=-\frac{1}{8 \pi^{2}}\left(\log \left(z_{1}\right)+2 z_{1}+240 z_{2}+3 z_{1}^{2}-480 z_{1} z_{2}+220680 z_{2}^{2}+\ldots\right),
$$

where

$$
z_{1}=\frac{1}{4 \phi^{2}}, \quad z_{2}=-\frac{2 \phi}{(12 \psi)^{6}} .
$$

This agrees with the conjectured relationship in [2]. One can show that the example with $h^{2,1}(Y)=3$ of [2] is also consistent with our statements. It is perhaps worth emphasizing that we have deduced (28) given only the existence of the weakly-coupled heterotic string - no further knowledge of the heterotic string (such as enhanced gauge symmetries) was required.

Let us now turn to the other $n_{\mathrm{v}}-1$ vector moduli. Now that we know that $X$ is a K3-fibration, we can say exactly where the other contributions to $h^{1,1}(X)$ come from. The general statement is that they can be divided into two classes - those from the generic fibre and those from the singular fibre. Let us concentrate first on the contribution from the generic fibre.

Let $C_{i}$ be an algebraic curve in a generic K3 fibre. As we move about the base space we can map the curve into equivalent curves in the other generic fibres. Adding all these curves together gives us a divisor, $D_{i}$ in $X$. That is, we have written $D_{i}$ as a fibration over $W$ where $C_{i}$ is the generic fibre. (Note that $D_{i}$ is not dual to $C_{i}$.) Thus, a curve in the generic fibre contributes to $h^{1,1}$. Because a generic K3-fibration has monodromy, we may have two curves in a generic fibre that can be mapped into each other by cycling round a path on $W$. Clearly such a pair only contribute one to $h^{1,1}$ as they both were required in building $D_{i}$.

Let us denote a generic fibre $K_{p}$, where $p \in W$. The set of (duals of) algebraic curves in $K_{p}$ generate a sublattice of $H^{2}\left(K_{p}, \mathbb{Z}\right)$ called the "Picard lattice" of $K_{p}$. Clearly both $H^{1,1}\left(K_{p}\right)$ and the lattice $H^{2}\left(K_{p}, \mathbb{Z}\right)$ can be embedded in $H^{2}\left(K_{p}, \mathbb{C}\right)$. The Picard lattice can be considered the intersection $H^{1,1}\left(K_{p}\right) \cap H^{2}\left(K_{p}, \mathbb{Z}\right)$.

Consider the space $H^{2}\left(K_{p}, \mathbb{R}\right)$. The natural wedge product between elements in this space endows it with a metric. One can show that this metric has signature $(3,19)$ (see, for example, [ [4]). Let $\omega$ be a $(2,0)$-form on $K_{p}$. One can show that the real and imaginary parts of $\omega$ span a space-like 2-plane in $H^{2}\left(K_{p}, \mathbb{R}\right) \cong \mathbb{R}^{3,19}$. As any element of the Picard lattice is a $(1,1)$-form and so lies orthogonal to this 2-plane, the Picard lattice must be embeddable in $\mathbb{R}^{1,19}$. The "Picard number" of a K3 surface is the rank of the Picard lattice. Clearly then, the Picard number can be no bigger than 20 .

The Kähler form on a K3 surface is a vector within the real space generated by the Picard lattice. The volume of the K3 surface is given by the length of this vector which must therefore be positive. Thus we have shown that the Picard lattice must have signature $(+,-,-, \ldots,-)$. From what we have said above, the vector multiplets coming from 
the generic fibre are the generators of the monodromy-invariant subspace of the Picard lattice. This subspace must contain the Kähler form of the fibre and so also has signature $(+,-,-, \ldots,-)$. The rank is clearly less than or equal to the Picard number.

Consider the intersection numbers $D_{s} \cdot D_{i} \cdot D_{j}$ where the latter two divisors come from curves in the generic fibres. This is equal to the intersection $C_{i} \cdot C_{j}$ within the fibre and is thus given by the intersection form of the monodromy-invariant Picard lattice. This means that we have exactly reproduced the heterotic string result (6) in terms of the type IIA string.

We also know that there is at least one (the volume of the fibre) but no more than 20 moduli from this source. Adding these to the dilaton and the $U(1)$ from the gravity multiplet shows that the rank of the gauge group for K3-fibrations with only contributions to $h^{1,1}$ from the base space and generic fibers satisfies

$$
3 \leq \operatorname{rank}\left(G_{I I}\right) \leq 22
$$

which is consistent with the heterotic bound (1). The upper limits are suspiciously close to each other and we can probably bring these results into agreement once we take into account the quantum geometry of the K3 fibre. In the above analysis we used purely classical geometry for the analysis of the Picard lattice. Given the appearance of quantum effects in K3 surfaces [ 45] it is not at all unreasonable to expect that some quantum Picard lattice exists for a Planck-sized K3 surface with Picard number 22. This would bring the upper limit of $G_{I I}$ into complete agreement with the heterotic limit. The lower limit of the heterotic vacuum can never be reached for a Calabi-Yau manifold since there necessarily has to be a volume form $J$ which obeys $J \cdot J \cdot J \neq 0$ and thus cannot be the image of the heterotic dilaton. However it is conceivable that a model which does not have a Calabi-Yau phase may be used to achieve this lower limit.

\section{Limitations of duality}

So far we have discussed the successes of the type IIA/heterotic duality picture. Now let us discuss some of the short-comings and the reasons why they must appear.

The first question which comes to mind concerns the contributions to $h^{1,1}(X)$ from the degenerate fibres. Let us call such a divisor $E$. Clearly such a divisor will not intersect the generic fibre $D_{s}$ and so

$$
D_{s} \cdot E \cdot E=0,
$$

in contradiction with (6) for the heterotic string. This implies that $K 3$-fibrations where $h^{1,1}(X)$ contains moduli corresponding to degenerate fibers cannot be the dual of a standard weakly coupled heterotic string vacuum as we described it in section 2. However, such fibrations still have a candidate for the image of the heterotic dilaton but there seem to be no heterotic states which $E$ can be the image of. If there are such states (31) implies that they 
decouple at the tree level and only arise at one-loop and/or nonperturbatively (equivalently this says that they cannot be states in the conformal field theory). Since in general $E \cdot E$. $E \neq 0$ the corresponding heterotic states would have to arise already at the one-loop level. Alternatively they could be states that become massless only nonperturbatively but with "unusual" couplings to the dilaton such as the Ramond-Ramond states in type II strings. Being members of vector multiplets they would enhance the rank of $G_{\text {het }}$ nonperturbatively but so far no such states have been identified directly in the heterotic string.

The number of states coming from singular fibres can be very large easily taking the rank of the gauge group beyond the expected limit of 24 . But as we have just argued these vector multiplets have no conformal field theory interpretations on the heterotic side and thus no contradiction arises. Conversely, demanding the absence of states corresponding to degenerate fibers implies the bound (30) perfectly consistent with the heterotic string.

What if $X$ is not a K3-fibration? What happens to the heterotic dual? This question is remarkably similar to the question of if a manifold has a mirror partner. A simple example of a manifold without a mirror partner is that of one with no deformations. This implies $h^{2,1}=0$ and so the mirror would have $h^{1,1}=0$ which is impossible for a Kähler manifold. It turns out however that even in many cases where $h^{2,1}>0$, the manifold may have no mirror. This was analyzed in [22] where it was shown that some $N=2$ superconformal field theories, appearing as the mirror of a Calabi-Yau theory, have a non-trivial moduli space of $(1,1)$-forms but nowhere in this moduli space is there a Calabi-Yau phase. ${ }^{8}$

We claim that the same thing happens for type II/heterotic duality. The moduli space of vector multiplets in a type IIA string may be non-trivial but it may be that there is simply no place in this moduli space where there is a weakly-coupled heterotic string. This should happen if $X$ is not a K3-fibration. Of course, this works both ways. Given the moduli space of vector multiplets of a heterotic string there may be no place in this space where a weakly-coupled type IIA string compactified on a Calabi-Yau manifold can be described.

It is worth emphasizing that missing dual partners can enter into the picture of connecting up the moduli space of $N=2$ theories via phase transitions as in [ 17, 17] and flops as in [ 48]. It may be that there is an extremal transition (such as a conifold transition) or flop ${ }^{9}$ on the type IIA side from a manifold which is a K3-fibration to a manifold which is not. In this case, the extremal transition or flop involves shrinking down the base $\mathbb{P}^{1}$ of the fibration. This means that on the heterotic side the phase transition occurs at strong coupling and the "new" phase no longer has a (heterotic) weak coupling limit.

\footnotetext{
${ }^{8}$ It is also possible that discrete torsion can lead to similar effects [46].

${ }^{9}$ We thank P. Berglund for discussions on this point.
} 


\section{Acknowledgements}

We thank M. Gross for many important contributions. It is also a pleasure to thank P. Argyres, P. Berglund, S. Chaudhuri, B. de Wit, B. Greene, J. Harvey, V. Kaplunovsky, D. Morrison, J. Polchinski, R. Schimmrigk and A. Strominger for useful conversations as well as all the other "Plancksters" at the I.T.P., Santa Barbara program where part of this work was done under the NSF grant PHY94-07194. We are especially grateful to P. Langacker, P. Nath and J. Polchinski for organizing a stimulating workshop. The work of P.S.A. is supported by a grant from the National Science Foundation. The work of J.L. is supported by a Heisenberg fellowship of the DFG and the German-Israeli foundation.

\section{References}

[1] N. Seiberg and E. Witten, Monopoles, Duality and Chiral Symmetry Breaking in N=2 Supersymmetric QCD, Nucl. Phys. B431 (1994) 484-550.

[2] S. Kachru and C. Vafa, Exact Results For N=2 Compactifications of Heterotic Strings, Nucl. Phys. B450 (1995) 69-89.

[3] S. Ferrara, J. Harvey, A. Strominger, and C. Vafa, Second Quantized Mirror Symmetry, CERN et al 1995 preprint CERN-TH 95/131, hep-th/9505162.

[4] C. Vafa and E. Witten, Dual String Pairs With $N=1$ and $N=2$ Supersymmetry in Four Dimensions, Harvard and IAS 1995 preprint HUTP-95/A023, hep-th/9507050.

[5] A. Sen and C. Vafa, Dual Pairs of Type II String Compactifications, Tata and Harvard 1995 preprint TIFR/TH/95-41, hep-th/9508064.

[6] V. Kaplunovsky, J. Louis, and S. Theisen, Aspects of Duality in N=2 String Vacua, Phys. Lett. 357B (1995) 71-75.

[7] A.Klemm, W.Lerche, and P.Mayr, K3-Fibrations and Heterotic-Type II String Duality, Phys. Lett. 357B (1995) 313-322.

[8] I. Antoniadis, E. Gava, K. S. Narain, and T. R. Taylor, N=2 Type II-Heterotic duality and Higher derivative F-terms, ICTP 1995 preprint IC/95/177, hep-th/9507115.

[9] I. Antoniadis and H. Partouche, Exact Monodromy Group of N=2 Heterotic Superstring, École Polytechnique 1995 preprint CPTH-RR370.0895, hep-th/9509009.

[10] M. Billó et al., A Search for Non-Perturbative Dualities of Local N=2 Yang-Mills Theories from Calabi-Yau Threefolds, CERN et al 1995 preprint CERN-TH 95/140, hep-th/9506075.

[11] G. L. Cardoso, D. Lüst, and T. Mohaupt, Non-Perturbative Monodromies in N=2 Heterotic String Vacua, Humboldt 1995 preprint HUB-IEP-95/12, hep-th/9507113. 
[12] S. Kachru et al., Nonperturbative Results on the Point Particle Limit of N=2 Heterotic String, Harvard 1995 preprint HUTP-95/A032, hep-th/9508155.

[13] C. Hull and P. Townsend, Unity of Superstring Dualities, Nucl. Phys. B438 (1995) 109-137.

[14] M. Duff, Strong/Weak Coupling Duality from the Dual String, Nucl.Phys. B442 (1995) 47-63.

[15] E. Witten, String Theory Dynamics in Various Dimensions, Nucl. Phys. B443 (1995) 85-126.

[16] A. Sen, String String Duality Conjecture in Six Dimensions and Charged Soliton Strings, Nucl. Phys. B450 (1995) 103-114.

[17] P. S. Aspinwall, An $N=2$ Dual Pair and a Phase Transition, Cornell 1995 preprint CLNS95/1366, hep-th/9510142.

[18] B. H. Lian and S.-T. Yau, Mirror Maps, Modular Relations and Hypergeometric Series I,II, Harvard 1995 preprint, hep-th/9507151, hep-th/9507153.

[19] G. Aldazabal, A. Font, L. E. Ibáñez, and F. Quevedo, Chains of N=2, D=4 Heterotic/Type II Duals, CERN et al 1995 preprint CERN-TH/95-270, hep-th/9510093.

[20] E. Witten, Phases of $N=2$ Theories in Two Dimensions, Nucl. Phys. B403 (1993) 159-222.

[21] P. S. Aspinwall, B. R. Greene, and D. R. Morrison, Calabi-Yau Moduli Space, Mirror Manifolds and Spacetime Topology Change in String Theory, Nucl. Phys. B416 (1994) 414-480.

[22] P. S. Aspinwall and B. R. Greene, On the Geometric Interpretation of $N=2$ Superconformal Theories, Nucl. Phys. B437 (1995) 205-230.

[23] T. Banks and L. Dixon, Constraints on String Vacua with Space-Time Supersymmetry, Nucl. Phys. B307 (1988) 93-108.

[24] B. de Wit, P. Lauwers, and A. Van Proeyen, Lagrangians of $N=2$ Supergravity-Matter Systems, Nucl. Phys. B255 (1985) 569-608.

[25] S. Ferrara, L. Girardello, C. Kounnas, and M. Porrati, Effective Lagrangians for FourDimensional Superstrings, Phys. Lett. 192B (1987) 368-376.

[26] S. Ferrara and A. Van Proeyen, A Theorem on N=2 Special Kähler Product Manifolds, Class. Quant. Grav. 6 (1989) L243-L247.

[27] A. Ceresole, R. D'Auria, S. Ferrara, and A. Van Proeyen, Duality Transformations in Supersymmetric Yang-Mills Theories Coupled to Supergravity, Nucl. Phys. B444 (1995) 92-124.

[28] B. de Wit, V. Kaplunovsky, J. Louis, and D. Lüst, Perturbative Couplings of Vector Multiplets in $N=2$ Heterotic String Vacua, Nucl.Phys. B451 (1995) 53-95.

[29] M. Bershadsky, S. Cecotti, H. Ooguri, and C. Vafa, Holomorphic Anomalies in Topological Field Theories, Nucl. Phys. B405 (1993) 279-304. 
[30] M. Bershadsky, S. Cecotti, H. Ooguri, and C. Vafa, Kodaira-Spencer Theory of Gravity and Exact Results for Quantum String Amplitudes, Commun. Math. Phys. 165 (1994) 311-428.

[31] I. Antoniadis, E. Gava, K. Narain, and T. Taylor, Topological Ampltudes in String Theory, Nucl. Phys. B413 (1994) 162-184.

[32] G. L. Cardoso and B. A. Ovrut, Coordinate and Kähler Sigma Model Anomalies and Their Cancelation in String Effective Field Theories, Nucl. Phys. B392 (1993) 315-344.

[33] L. E. Ibáñez and D. Lüst, Duality Anomaly Cancellation, Minimal String Unification and the Effective Low-Energy Lagrangian of 4-d Strings, Nucl. Phys. B382 (1992) 305-364.

[34] I. Antoniadis, E. Gava, and K. Narain, Moduli Corrections to Gauge and Gravitational Couplings in Four-Dimensional Superstrings, Nucl. Phys. B383 (1992) 93-109.

[35] L. J. Dixon, V. Kaplunovsky, and C. Vafa, On Four-Dimensional Gauge Theories from Type-II Superstrings, Nucl. Phys. B294 (1987) 43-82.

[36] M. Dine, N. Seiberg, X. G. Wen, and E. Witten, Nonperturbative Effects on the String WorldSheet, Nucl. Phys. B278 (1986) 769-789, and Nucl. Phys. B289 (1987) 319-363.

[37] P. S. Aspinwall and D. R. Morrison, Topological Field Theory and Rational Curves, Commun. Math. Phys. 151 (1993) 245-262.

[38] P. S. Aspinwall, B. R. Greene, and D. R. Morrison, Measuring Small Distances in $N=2$ Sigma Models, Nucl. Phys. B420 (1994) 184-242.

[39] K. Oguiso, On Algebraic Fiber Space Structures on a Calabi-Yau 3-fold, Int. J. of Math. 4 (1993) 439-465.

[40] B. R. Greene and M. R. Plesser, Duality in Calabi-Yau Moduli Space, Nucl. Phys. B338 (1990) 15-37.

[41] P. S. Aspinwall, B. R. Greene, and D. R. Morrison, The Monomial-Divisor Mirror Map, Internat. Math. Res. Notices 1993 319-338.

[42] P. Candelas et al., Mirror Symmetry for Two Parameter Models - I, Nucl. Phys. B416 (1994) 481-562.

[43] S. Hosono, A. Klemm, S. Theisen, and S.-T. Yau, Mirror Symmetry, Mirror Map and Applications to Calabi-Yau Hypersurfaces, Nucl. Phys. B433 (1995) 501-554.

[44] W. Barth, C. Peters, and A. van de Ven, Compact Complex Surfaces, Springer, 1984.

[45] P. S. Aspinwall and D. R. Morrison, String Theory on K3 Surfaces, Duke and IAS 1994 preprint DUK-TH-94-68, IASSNS-HEP-94/23, hep-th/9404151, to appear in "Essays on Mirror Manifolds 2". 
[46] P. Berglund, Dimensionally Reduced Landau-Ginzburg Orbifolds with Discrete Torsion, Phys. Lett. 319B (1993) 117-124.

[47] B. R. Greene, D. R. Morrison, and A. Strominger, Black Hole Condensation and the Unification of String Vacua, Nucl. Phys. B451 (1995) 109-120.

[48] P. S. Aspinwall, B. R. Greene, and D. R. Morrison, Multiple Mirror Manifolds and Topology Change in String Theory, Phys. Lett. 303B (1993) 249-259. 